\title{
Continuity effects on rotifers and microcrustaceans caused by the construction of a downstream reservoir in a cascade series (Iguaçu River, Brazil)
}

\author{
M. Serafim-Júniora*, F. A. Lansac-Tôha ${ }^{b}$, R. M. Lopes ${ }^{c}$ and G. Perbiche-Neves ${ }^{d}$ \\ ${ }^{a}$ Curso de Engenharia de Pesca, Laboratório de Aquicultura, Universidade Federal do Recôncavo da Bahia - UFRB, \\ Campus Universitário de Cruz das Almas, CEP 44380-000, Cruz das Almas, BA, Brazil \\ bNúcleo de Estudos em Limnologia, Ictiologia e Aquicultura - NUPÉLIA, Laboratório de Zooplâncton, \\ Universidade Estadual de Maringá - UEM, Avenida Colombo, 5790, CEP 87020-900, Maringá, PR, Brazil \\ 'Laboratório de Sistemas Planctônicos, Departamento de Oceanografia Biológica, Instituto Oceanográfico, \\ Universidade de São Paulo - USP, Praça do Oceanográfico, 191, Cidade Universitária, CEP 05508-120, São Paulo, SP, Brazil \\ ${ }^{\mathrm{d} C e n t r o ~ d e ~ C i e ̂ n c i a s ~ d a ~ N a t u r e z a, ~ U n i v e r s i d a d e ~ F e d e r a l ~ d e ~ S a ̃ o ~ C a r l o s ~-~ U F S C a r, ~ C a m p u s ~ L a g o a ~ d o ~ S i n o, ~}$ \\ Rodovia Lauri Simões de Barros, Km 12 - SP 189, Bairro Aracaçú, CP 094, CEP 18290-000, Buri, SP, Brazil \\ *e-mail: m.serafim@ufrb.edu.br
}

Received: January 17, 2014 - Accepted: January 15, 2015 - Distributed: May 31, 2016

(With 4 figures)

\begin{abstract}
This study evaluated changes in the community of rotifers and microcrustaceans associated with the construction of a large and dendritic reservoir in Iguaçu River (Brazil), as the last reservoir of a sequence of five cascading systems. Differences were clear between pre-filling and post-filling phases for organisms and some of environmental variables. In the pre-filling phase, the community was more homogeneous along the downstream river gradient, and spatial compartmentalization in the new reservoir was common during the post-filling phase. From 140 identified taxa, 10 species occurred exclusively in the pre-filling phase and 32 in the post-filling phase. After completion of the fifth reservoir filling-up, opportunistic, pioneer and fast-developing species quickly dominated, and downstream of the fourth reservoir the pre-filling decreasing gradient of richness, diversity and evenness disappeared. Richness of rotifers and cladocerans, cladocerans diversity, and evenness of rotifers, cladocerans and copepods were generally higher in the post-filling phase. A non-metric multidimensional analysis based on a presence/absence matrix depicted a homogeneous and dense group of species associated to the pre-filling phase and a second, dispersed group related to the post-filling phase. Spearman correlations pointed out significant positive effects of transparency on rotifer species richness in the post-filling phase, and negative effects on the microcrustacean richness in the pre-filling phase. Dam construction caused disruption of the downstream lotic gradient along the series of dams, leading to the development of distinct species in lentic spatial compartments.
\end{abstract}

Keywords: Rotifera, Cladocera, Copepoda, Iguaçu River.

\section{Efeitos contínuos sobre rotíferos e microcrustáceos causados pela construção de um reservatório a jusante de uma série em cascata, Rio Iguaçú, Brasil}

\begin{abstract}
Resumo
Esse estudo analisou os efeitos da construção de um reservatório largo e dendrítico no Brasil sobre a comunidade de rotíferos e microcrustáceos, sendo o último reservatório de uma cascata de cinco. Houve claras diferenças entre as fases pré e pós-enchimento para os organismos e para algumas variáveis ambientais. Na fase pré-enchimento, a comunidade foi mais homogênea no gradiente longitudinal no rio a jusante, e na fase pós-enchimento houve compartimentalização espacial no novo reservatório. Dos 140 táxons identificados, dez espécies ocorreram exclusivamente na fase pré-enchimento e 32 na fase pós-enchimento. Após o enchimento, espécies oportunistas, pioneiras e de rápido desenvolvimento foram dominantes, e desapareceu o gradiente de decréscimo a jusante do quarto reservatório para a riqueza, diversidade e equitabilidade durante a fase pré-enchimento. A riqueza de rotíferos e cladóceros, a diversidade de cladóceros, e a equitabilidade de rotíferos, cladóceros e copépodes foram elevadas na fase pós-enchimento. A análise de escalonamento multidimensional não-métrico sobre a matriz de presença e ausência mostrou um grupo homogêneo e denso de espécies e meses de amostragem na fase pré-enchimento, e outro grupo disperso na fase pós-enchimento. Correlações de Spearman apontaram efeitos significativos da transparência sobre a riqueza de rotíferos na fase pós-enchimento, e efeitos negativos sobre a riqueza de microcrustáceos na outra fase. Os efeitos da construção do reservatório causaram rompimento no gradiente lótico contínuo a jusante do quarto reservatório observado na fase pré-enchimento, permitindo o desenvolvimento de espécies em compartimentos espaciais lênticos.
\end{abstract}

Palavras-chave: Rotifera, Cladocera, Copepoda, Rio Iguaçu. 


\section{Introduction}

The damming of rivers leads to significant changes in the abiotic environment and in the biological communities both upstream and downstream (Marzolf, 1990; Straskraba and Tundisi, 1999; Garrido and Bozelli, 2000; Ribeiro Filho et al., 2011). Changes in abiotic conditions are induced by reduction of water velocity, increase of water transparency, and the possibility of formation of thermal and chemical stratification. For plankton communities, changes occur in several ecological attributes such as species richness, diversity and abundance of phytoplankton and zooplankton, when lotic species are replaced by lentic species after stabilisation of the system (Pedrozo et al., 2012). The interactions occurring between the river basin and the impounded water lead to the establishment of spatial gradients (longitudinal and lateral gradients) of biotic and abiotic variables in the reservoir main channel (Perbiche-Neves and Nogueira, 2010; Perbiche-Neves et al., 2011). The formation of many side-arms generally associated with tributary rivers is common in dendritic reservoirs, which can also contribute to the formation of lateral gradients (Ward, 1989; Van Den Brink et al., 1994; Bonecker et al., 2001).

In reservoirs, zooplankton communities exhibit structural changes along longitudinal and lateral gradients, because of the interaction with environmental factors such as water flow, physical and chemical stratification, and tributary entrances (Nogueira, 2001; Sampaio et al., 2002; Sartori et al., 2009). Higher densities are expected to occur in transition zones, both between the main river and the reservoir and between tributaries and the reservoir (backwater areas) (Marzolf, 1990; Van Den Brink et al., 1994). In cascade series of reservoirs, the upstream reservoirs - especially those of the storage type with a high retention time - affect the zooplankton downstream (Sampaio et al., 2002; Nogueira et al., 2008; Perbiche-Neves and Nogueira, 2010, 2013), exporting fauna by drifting process (Mitsuka and Henry, 2002; Perbiche-Neves et al., 2012). The organisms tend to disperse downstream, but construction of a new dam can stop this process due to the development of lentic conditions. Although there are many reservoir cascades in the world, the effects of a new impoundment at the end of a series of reservoirs have rarely been studied.

After the filling of a new reservoir, aquatic communities tend to follow a continuous process of changes and succession (Garrido and Bozelli, 2000; Pedrozo et al., 2012). In the initial years after impoundment, irregular reproduction cycles for many species of zooplankton occur (Perbiche-Neves et al., 2007; Serafim-Júnior et al., 2010). Colonisation processes range from $r$ - to $k$-strategist species, initially with the dominance of small species (including rotifers, protozoans, small cladocerans and cyclopoid copepods), which tend to be replaced by large species, such as large cladocerans and calanoid copepods (Matsumura-Tundisi et al., 1991; Santos, 1995; Bonecker et al., 2001; Pedrozo et al., 2012). However, in the Neotropical region, comparative studies on zooplankton community in the pre- and post-impoundment phases remain scarce (Lansac-Tôha et al., 1999; Bonecker et al., 2001; Velho et al., 2001; Pedrozo et al., 2012), and additional investigations are needed to understand the succession mechanisms along the different filling stages.

In this study, we examined the composition, richness, alpha diversity and evenness of rotifers and microcrustaceans in relation to environmental variables, comparing a year before and a year after the construction of a large and dendritic reservoir in the Iguaçu River, south of Brazil. We tested two hypotheses: 1) that the decreasing gradient downstream from the last reservoir will disappear after damming of the new reservoir, due to the establishment of lentic conditions favourable to some rotifers and microcrustaceans, such as increase in water transparency and chlorophyll; 2) that richness, diversity and evenness will increase in the post-filling phase, for the same reasons.

\section{Material and Methods}

The study was carried out in the low stretch of the Iguaçu River, in the Paraná State, south of Brazil. The climate in this region is Cfa sub-tropical (Koppen), usually with two distinct seasons: cool-dry (winter) and warm-wet (summer). The mean annual rainfall ranges from 1,500 to $1,700 \mathrm{~mm}$.

The studied reservoir (Figure 1), named Salto Caxias

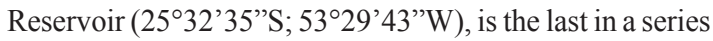
of five large reservoirs in this river, all used for hydropower generation. The series of reservoirs along Iguaçu River is: Foz do Areia, Segredo, Salto Santiago, Salto Osório and Salto Caxias. A sixth new reservoir (Capanema) is being built in Iguaçu River few kilometres downstream Salto Caxias dam. The Salto Caxias Reservoir construction was completed in September of 1998 and is located in the final stretch of the Iguaçu River. The lake has a surface area of $141 \mathrm{~km}^{2}$ with an annual dam discharge of $3.6 \times 10^{6} \mathrm{~m}^{3}$. The mean water retention time of the lake is 38 days, the mean depth is $40 \mathrm{~m}$ and the maximum is $60 \mathrm{~m}$ close to the dam.

For this study, a total of nine sampling sites were selected along the Iguaçu River stretch and its tributaries (Figure 1). The organisms were sampled monthly, from March of 1997 to February of 1998 (pre-impoundment phase) and from April of 1999 to March of 2000 (post-impoundment phase), totalling 216 samples. Samples were collected by filtering $300 \mathrm{~L}$ of water of sub-surface through $64-\mu \mathrm{m}$ plankton net, with the aid of a motorised pump Stihl P835 and flow of $40 \mathrm{~L} / \mathrm{h}$. Organisms were immediately preserved in $4 \%$ buffered formalin. For microscopic analysis, sample aliquots of $1 \mathrm{~mL}$ were obtained using Hensen-Stempel pipettes. Bdelloidea rotifers and Harpacticoida copepods, as well as nauplii and copepodites of Cyclopoida and Calanoida, were not identified to lower taxonomic level. Rotifers and copepod nauplii were analysed in Sedgwick-Rafter chambers and adult cladocerans and adult and copepodites of copepods in gridded acrylic chambers, under an optical microscope and stereomicroscope, respectively. 


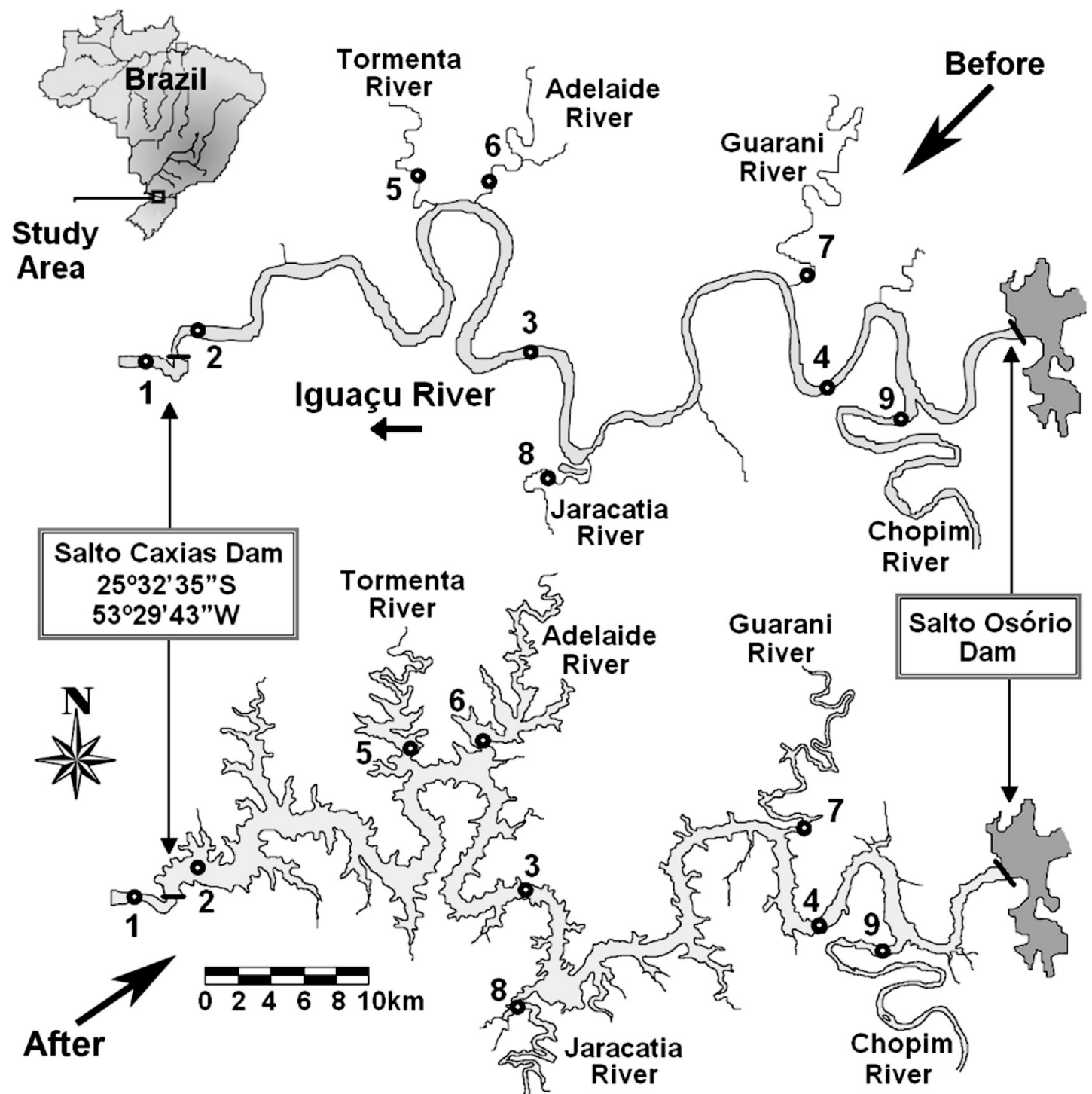

Figure 1. Map of study area and sampling sites in the pre-filling phase and post-filling phase of the Iguaçu River, at the Salto Caxias Reservoir.

Species of rotifers, copepods and cladocerans were classified as their habits in planktonic or non-planktonic, according to Koste (1978), Segers (1995), Lima et al. (1998), and Serafim-Júnior et al. (2003).

The frequency of occurrence of each taxon was calculated by considering the numbers of samples in which the species were found in relation to the total number of samples. Species richness was determined according to the number of species recorded in each sample. Individual abundance and the Shannon-Wiener diversity index $\left(H^{\prime}=\Sigma\right.$ pi. $\left.\log _{2} \cdot p_{i}\right)$, where pi is the proportion of taxon ${ }_{i}$ in the sample, and evenness ( $\left.\mathrm{E}=\mathrm{H}^{\prime} / \mathrm{H}^{\prime} \max \right)$ (Pielou, 1984) were also determined.

Data of richness, diversity and evenness were examined by a factorial analysis of variance (ANOVA), using as factors the filling phases (pre- and post-filling), sampling sites, and the interaction between both. ANOVA detected the effects of phases on sampling sites, using the significant differences in the interaction to represent the changes in ecological attributes. The assumptions of normality and homoscedasticity were previously checked with Shapiro-Wilk test and Levene test, respectively. The analysis was run in Statistica 6.0 (STATSOFT INC, 2002).

The VEGAN and MASS packages in the software $\mathrm{R}$ Cran Project (R CORE DEVELOPMENT TEAM, 2012) were used for non-metric multidimensional analysis (NMDS, using isoMDS) to separate species and sampling months between pre- and post-filling phases, based on a presence/absence matrix. First, all data of sampling sites and months were analysed, but due to the excessive number of lines we opted to estimate a mean among sampling sites for each sampling date, for the final analyses. Bray-Curtis 
distance was used. The convergent solutions were found by several trials. For scaling, centring, PC rotation and half change scaling were used.

Eleven environmental variables were measured in each sampling site, including: temperature, electrical conductivity, dissolved oxygen and $\mathrm{pH}$ (Horiba U10 probe), transparency (Secchi disk), chlorophyll (Talling and Driver, 1963), alkalinity total phosphorus (Strickland and Parsons, 1960), total nitrogen (Mackreth et al., 1978), total solids (TS) and total suspended solids (TSS). In the pre-filling phase limnological variables were measured in the water surface, and in the post-filling phase water samples were taken with a Van Dorn bottle in several depths, selected according to transparency, values. Sonde measurements were made on board immediately after sampling, and sample jars were frozen subsequently for later chemical analysis. Mean values of each limnological variable were calculated for the entire water column for statistical analysis. One-way ANOVA was performed to test for significant differences in limnological variables between pre- and post-filling phases. Spearman correlations were estimated between environmental variables and rotifer and species richness in pre- and post-filling phases, in order to distinguish environmental influences on rotifers and microcrustaceans richness. Statistical analyses were performed on Statistica 6.0 (STATSOFT INC, 2002).

\section{Results}

A total of 140 taxa were identified in both phases, 10 of which were only recorded in the pre-impoundment phase, and 32 only in the post-impoundment phase. Most species are shown in Table 1 (except very rare species which were not used in all statistical analysis). We found 99 species of rotifers distributed in 20 families. Eight species of rotifers were only found in the pre-impoundment phase and 21 in the post-impoundment phase. The most common rotifers in the pre-impoundment phase were Keratella cochlearis (61\%), Polyarthra vulgaris (61\%), K. americana (51\%), Conochilus coenobasis (44\%) and
Ploesoma truncatum (43\%). In the post-impoundment phase, Polyarthra vulgaris $(89 \%), K$. cochlearis $(84 \%)$, C. coenobasis (83\%), C. unicornis (82\%), Collotheca sp. (81\%), K. americana (80\%) and Hexarthra intermedia brasiliensis $(74 \%)$ were common.

Among the microcrustaceans, 26 species of cladocerans and 15 species of copepods were identified, the latter comprised by seven species of Cyclopidae and eight species of Diaptomidae.

Moina reticulata was only observed in the Iguaçu River (site 3) during the pre-impoundment phase. In contrast, seven species of Cladocera were exclusive to the post-impoundment phase. The most common cladocerans in the pre-impoundment phase were Ceriodaphnia cornuta (32\%), Daphnia gessneri (27\%) and Bosmina hagmanni $(25 \%)$. In the post-impoundment phase, Moina minuta $(80 \%)$ was the most common, followed by B. hagmanni (78\%), C. cornuta (66\%) and Bosminopsis deitersi (63\%).

Thermocyclops decipiens (41\%) and Argyrodiaptomus furcatus $(27 \%)$ were dominant in the pre-impoundment phase. In the post-impoundment phase, the dominant species were $T$. decipiens (58\%), followed by Microcyclops anceps (36\%), Notodiaptomus henseni (25\%), N. anisitsi (22\%) and $N$. deitersi (22\%). Five species of copepods were only found in the post-impoundment phase. Significant differences for important ecological attributes were detected between the pre- and post-filling phases, for rotifers, cladocerans and copepods (Table 2). The interaction between phases and sites was significant for richness and evenness of rotifers, for evenness of copepods, and for all attributes of cladocerans (Table 1). Among the sampling sites, only the evenness of rotifers was not different. Rotifer richness was usually high in the post-filling phase (Figure 2a) when differences among sampling sites were minor; the opposite was observed in the pre-filling phase, when low rotifer richness occurred at the tributaries. Rotifer evenness was higher in the pre-filling phase and in tributaries; during the post-filling phase evenness increased in the main channel (Figure 3b).
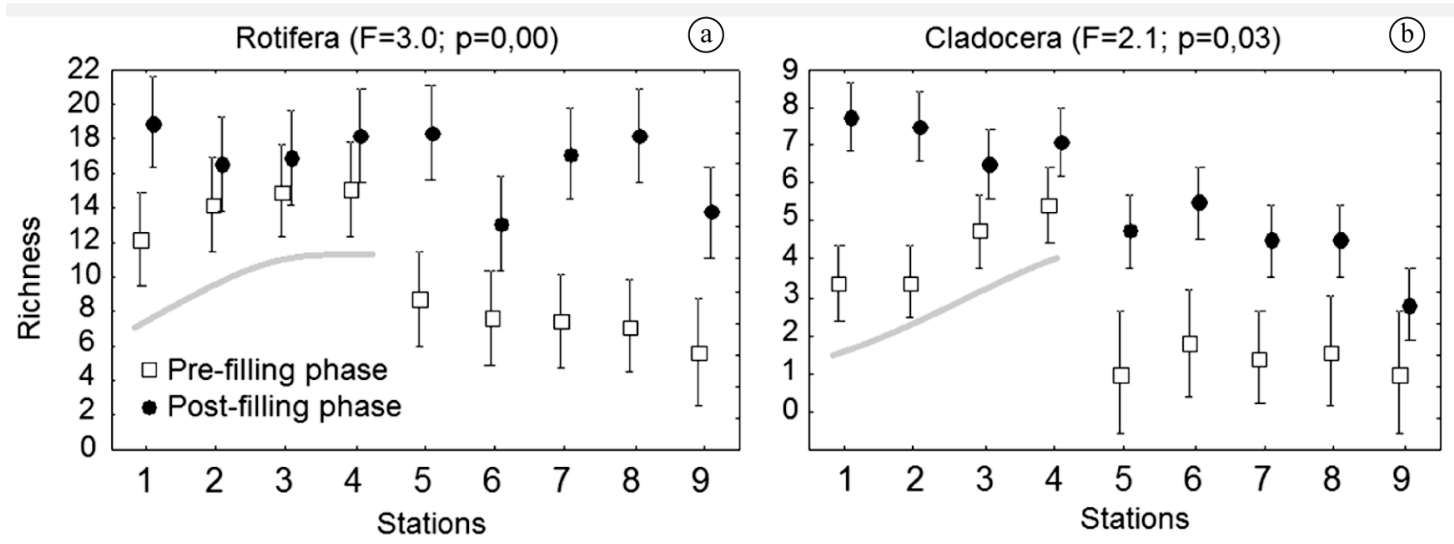

Figure 2. Mean values and standard error for richness of rotifers (a) and cladocerans (b), which showed significant interactions in the bifactorial ANOVA. 
Table 1. List of almost all species found in our study, with abbreviations used in NMDS and habitat classification (C) in planktonic (pk) or non-planktonic (npk). Species not identified in species level were not classified.

\begin{tabular}{|c|c|c|c|c|c|}
\hline Rotifera & Ab. & $\mathbf{C}$ & Rotifera & Ab. & $\mathbf{C}$ \\
\hline $\begin{array}{l}\text { Anuraeopsis naviculata } \\
\text { Rousselet, } 1910\end{array}$ & Anav & pk & $\begin{array}{c}\text { Platyas quadricornis } \\
\text { (Ehrenberg, 1832) }\end{array}$ & Pqua & $\mathrm{pk}$ \\
\hline $\begin{array}{l}\text { Ascomorpha ecaudis } \\
\text { (Perty, 1859) }\end{array}$ & Aeca & npk & $\begin{array}{c}\text { Platyias quadricornis } \\
\text { brevispina (Daday, 1905) }\end{array}$ & Pbrev & $\mathrm{pk}$ \\
\hline $\begin{array}{l}\text { Ascomorpha ovalis } \\
\text { (Bergendal, 1892) }\end{array}$ & Aova & npk & $\begin{array}{c}\text { Platyonus patulus } \\
\text { (O. F. Müller, 1786) }\end{array}$ & Plpat & npk \\
\hline $\begin{array}{l}\text { Ascomorpha saltans } \\
\text { Bartsch, } 1870\end{array}$ & Asal & pk & $\begin{array}{l}\text { Platyonus patulus var. } \\
\text { macracanthus } \\
\text { (Daday, 1905) }\end{array}$ & Plmac & npk \\
\hline $\begin{array}{l}\text { Asplanchna sieboldi } \\
\text { (Leydig, 1854) }\end{array}$ & Asieb & $\mathrm{pk}$ & $\begin{array}{c}\text { Ploesoma truncatum } \\
\text { (Levander, 1894) }\end{array}$ & Ptrun & $\mathrm{pk}$ \\
\hline $\begin{array}{l}\text { Brachionus angularis } \\
\text { Gosse, } 1851\end{array}$ & Bang & pk & $\begin{array}{c}\text { Polyarthra dolichoptera } \\
\text { (Idelson, 1925) }\end{array}$ & Pdoli & $\mathrm{pk}$ \\
\hline $\begin{array}{l}\text { Brachionus budapestinensis } \\
\text { Daday, } 1885\end{array}$ & Bbud & $\mathrm{pk}$ & $\begin{array}{c}\text { Polyarthra remata } \\
\text { (Skorikov, 1896) }\end{array}$ & Prem & npk \\
\hline $\begin{array}{l}\text { Brachionus calyciflorus } \\
\text { Pallas, } 1766\end{array}$ & Bcal & $\mathrm{pk}$ & $\begin{array}{c}\text { Polyarthra vulgaris } \\
\text { (Carlin, 1943) }\end{array}$ & Pvul & $\mathrm{pk}$ \\
\hline $\begin{array}{l}\text { Brachionus caudatus } \\
\text { insuetus Ahlstrom, } 1940\end{array}$ & Bins & npk & $\begin{array}{c}\text { Pompholyx complanata } \\
\text { Gosse, } 1851\end{array}$ & Pcom & npk \\
\hline $\begin{array}{l}\text { Brachionus caudatus } \\
\text { personatus Ahlstrm, } 1940\end{array}$ & Bpers & pk & Ptygura sp. & Ptysp & \\
\hline $\begin{array}{l}\text { Brachionus falcatus } \\
\text { falcatus Zacharias, } 1898\end{array}$ & Bfal & $\mathrm{pk}$ & $\begin{array}{l}\text { Scaridium longicaudatum } \\
\text { (O. F. Müller, 1786) }\end{array}$ & Scari & npk \\
\hline $\begin{array}{l}\text { Brachionus quadridentatus } \\
\text { (Hermanns, 1783) }\end{array}$ & Bqua & pk & Synchaeta sp.1 & Sysp1 & \\
\hline $\begin{array}{l}\text { Brachionus mirus reductus } \\
\text { (Koste, 1972) }\end{array}$ & Bred & pk & Synchaeta sp.2 & Sysp2 & \\
\hline $\begin{array}{l}\text { Cephalodella gibba } \\
\text { (Ehrenberg, 1838) }\end{array}$ & Cgib & npk & $\begin{array}{c}\text { Testudinella ahlstromi } \\
\text { (Hauer, 1956) }\end{array}$ & Tahls & npk \\
\hline $\begin{array}{l}\text { Cephalodella stenrossi } \\
\text { Wulfert, } 1937\end{array}$ & Cste & npk & $\begin{array}{c}\text { Testudinella patina patina } \\
\text { (Hermann, 1783) }\end{array}$ & Tpat & npk \\
\hline Collotheca sp. & Cosp & & $\begin{array}{l}\text { Trichocerca }(D) \text { similis } \\
\text { grandis (Hauer, 1965) }\end{array}$ & Tsim & $\mathrm{pk}$ \\
\hline Collotheca sp. & Csp & & $\begin{array}{c}\text { Trichocerca capucina } \\
\text { Wierzejski \& Zacharias, } \\
1893\end{array}$ & Tcap & $\mathrm{pk}$ \\
\hline $\begin{array}{l}\text { Conochilus coenobasis } \\
\text { Skorikov, } 1914\end{array}$ & Cocoe & npk & $\begin{array}{c}\text { Trichocerca cylindrica } \\
\text { chattoni } \\
\text { De Beauchamp, } 1907\end{array}$ & Tchat & $\mathrm{pk}$ \\
\hline $\begin{array}{l}\text { Conochilus unicornis } \\
\text { Rousselet, } 1892\end{array}$ & Couni & npk & $\begin{array}{c}\text { Trichocerca fusiformis } \\
\text { Levander, } 1894\end{array}$ & Tfusi & $\mathrm{pk}$ \\
\hline $\begin{array}{l}\text { Corulella obtusa } \\
\text { (Gosse, 1886) }\end{array}$ & Cobt & npk & $\begin{array}{c}\text { Trichocerca haueroensis } \\
\text { (Gillard, 1967) }\end{array}$ & Tmucr & $\mathrm{pk}$ \\
\hline $\begin{array}{l}\text { Dicranophorus epicharis } \\
\text { Harring \& Myers, } 1928\end{array}$ & Depic & npk & $\begin{array}{c}\text { Trichocerca insignis } \\
\text { (Lauterborn, 1898) }\end{array}$ & Tins & $\mathrm{pk}$ \\
\hline $\begin{array}{l}\text { Dicranophorus caudatus } \\
\text { brasiliensis Koste, } 1972\end{array}$ & Dcbra & $\mathrm{pk}$ & $\begin{array}{c}\text { Trichocerca parvula } \\
\text { Carlin, } 1939\end{array}$ & Tparv & npk \\
\hline $\begin{array}{l}\text { Dipleuchlanis propatula } \\
\text { (Gosse, 1886) }\end{array}$ & Dipro & npk & $\begin{array}{l}\text { Trichocercapusilla } \\
\text { (Lauterborn, 1898) }\end{array}$ & Tpus & $\mathrm{pk}$ \\
\hline Encentrum sp. & Encsp & & Trichocerca sp. & Trisp & \\
\hline $\begin{array}{l}\text { Epiphanes clavatula } \\
\text { (Ehrenberg, 1832) }\end{array}$ & Epcla & npk & $\begin{array}{c}\text { Trichocerca rousseleti } \\
\text { (Voigt, 1902) }\end{array}$ & Trous & $\mathrm{pk}$ \\
\hline
\end{tabular}


Table 1. Continued...

\begin{tabular}{|c|c|c|c|c|c|}
\hline Rotifera & Ab. & $\mathbf{C}$ & Rotifera & Ab. & $\mathbf{C}$ \\
\hline $\begin{array}{l}\text { Euchlanis dilatata } \\
\text { Ehrenberg, } 1832\end{array}$ & Edil & npk & $\begin{array}{l}\text { Trichotria tetractis } \\
\text { (Ehrenberg, 1830) }\end{array}$ & Trtetr & npk \\
\hline $\begin{array}{l}\text { Euchlanis incisa incisa } \\
\text { Carlin, } 1939\end{array}$ & Einc & npk & $\begin{array}{c}\text { Trichocerca scipio } \\
\text { Pejler, } 1962 \\
\end{array}$ & Tsci & $\mathrm{pk}$ \\
\hline $\begin{array}{l}\text { Euchlanis incisa mucronata } \\
\text { (Ahlstrom, 1934) }\end{array}$ & Emuc & npk & Cladocera & & \\
\hline $\begin{array}{l}\text { Filinia longiseta } \\
\text { (Ehrenberg, 1834) }\end{array}$ & Flon & $\mathrm{pk}$ & $\begin{array}{c}\text { Alona fasciculata } \\
\text { Daday, } 1905\end{array}$ & Afasc & npk \\
\hline $\begin{array}{l}\text { Gastropus hyptopus } \\
\text { Ehrenberg, } 1838\end{array}$ & Ghyp & $\mathrm{pk}$ & Alona guttata Sars, 1862 & Agut & npk \\
\hline $\begin{array}{l}\text { Hexarthra intermedia } \\
\text { brasiliensis (Hauer 1953) }\end{array}$ & Hbra & $\mathrm{pk}$ & $\begin{array}{c}\text { Alona intermedia } \\
\text { Sars, } 1862 \\
\end{array}$ & Ainte & npk \\
\hline $\begin{array}{l}\text { Hexarthra mira } \\
\text { (Hudson, 1871) }\end{array}$ & Hmira & pk & $\begin{array}{c}\text { Bosmina freyi } \\
\text { De Melo \& Hebert, } 1994\end{array}$ & Blong & pk \\
\hline $\begin{array}{l}\text { Horaella thomasoni } \\
\text { Koste, } 1973\end{array}$ & Htho & $\mathrm{pk}$ & $\begin{array}{c}\text { Bosmina hagmanni } \\
\text { Stingelin, } 1904\end{array}$ & Bhag & pk \\
\hline $\begin{array}{l}\text { Kellicottia bostoniensis } \\
\text { (Rousselet, 1908) }\end{array}$ & Kbost & pk & $\begin{array}{c}\text { Bosmina haueronensis } \\
\text { Delachaux, } 1918\end{array}$ & Bhaue & pk \\
\hline $\begin{array}{l}\text { Keratella americana } \\
\text { Carlin, } 1943\end{array}$ & Kame & pk & $\begin{array}{c}\text { Bosmina tubicen } \\
\text { Brehm, } 1939 \\
\end{array}$ & Btub & pk \\
\hline $\begin{array}{l}\text { Keratella cochlearis } \\
\text { Gosse, } 1851\end{array}$ & Kcoc & pk & $\begin{array}{c}\text { Bosminopsis deitersi } \\
\text { Richard, } 1834 \\
\end{array}$ & Bdei & pk \\
\hline $\begin{array}{l}\text { Keratella lenzi } \\
\text { (Hauer, 1953) }\end{array}$ & Klen & pk & $\begin{array}{c}\text { Ceriodaphnia cornuta } \\
\text { Sars, } 1886 \\
\end{array}$ & Ccor & pk \\
\hline $\begin{array}{l}\text { Keratella tropica } \\
\text { (Apstein, 1907) }\end{array}$ & Ktro & $\mathrm{pk}$ & $\begin{array}{l}\text { Ceriodaphnia cornuta } \\
\text { rigaudi }\end{array}$ & Ccorr & $\mathrm{pk}$ \\
\hline Lancinularia sp. & Lansp & & Ceriodaphnia sp. & Cersp & \\
\hline Lecane bulla (Gosse, 1851) & Lbul & npk & $\begin{array}{c}\text { Chydorus eurynotus } \\
\text { Sars, } 1901\end{array}$ & Ceur & npk \\
\hline $\begin{array}{l}\text { Lecane closterocerca } \\
\text { (Schmarda, 1859) }\end{array}$ & Lclos & npk & $\begin{array}{c}\text { Chydorus pubescens } \\
\text { Sars, } 1901 \\
\end{array}$ & Cpub & npk \\
\hline $\begin{array}{l}\text { Lecane cornuta } \\
\text { (Müller, 1786) }\end{array}$ & Lcor & npk & $\begin{array}{c}\text { Coronatella monacantha } \\
\text { Sars (1901) }\end{array}$ & Cmon & npk \\
\hline $\begin{array}{l}\text { Lecane curvicornis } \\
\text { (Murray, 1913) }\end{array}$ & Lcur & npk & $\begin{array}{c}\text { Daphnia gessneri Herbst, } \\
1967\end{array}$ & Dges & $\mathrm{pk}$ \\
\hline $\begin{array}{l}\text { Lecane flexvile } \\
\text { (Gosse, 1886) }\end{array}$ & Lfle & npk & $\begin{array}{c}\text { Daphnia parvula } \\
\text { (Fordyce, 1905) }\end{array}$ & Dpar & $\mathrm{pk}$ \\
\hline $\begin{array}{l}\text { Lecane halyclista } \\
\text { Harring \& Myers, } 1926\end{array}$ & Lhaly & $\mathrm{npk}$ & $\begin{array}{c}\text { Diaphanosoma birgei } \\
\text { Korineck } 1981\end{array}$ & Dbirg & $\mathrm{pk}$ \\
\hline $\begin{array}{l}\text { Lecane hamata } \\
\text { (Stokes, 1896) }\end{array}$ & Lham & npk & $\begin{array}{c}\text { Diaphanosoma brevireme } \\
\text { Sars } 1901\end{array}$ & Dbrev & npk \\
\hline $\begin{array}{l}\text { Lecane hornemanni } \\
\text { (Ehrenberg, 1834) }\end{array}$ & Lhorn & $\mathrm{npk}$ & $\begin{array}{c}\text { Guernella rafaelis } \\
\text { Richard, } 1892\end{array}$ & Graf & npk \\
\hline $\begin{array}{l}\text { Lecane leontina } \\
\text { (Turner, 1892) }\end{array}$ & Lleo & npk & $\begin{array}{c}\text { Ilyocryptus spinifer } \\
\text { Herrick, } 1882\end{array}$ & Ispinr & npk \\
\hline $\begin{array}{l}\text { Lecane ludwigii } \\
\text { (Eckstein, 1883) }\end{array}$ & Lich & npk & Leydigia sp. & Leyd & \\
\hline $\begin{array}{l}\text { Lecane ludwigii f. } \\
\text { laticaudata (Herrick, 1885) }\end{array}$ & Llud & $\mathrm{npk}$ & $\begin{array}{c}\text { Macrothrix spinosa } \\
\text { King, } 1853\end{array}$ & Maspi & npk \\
\hline $\begin{array}{l}\text { Lecane luna } \\
\text { (O. F. Müller, 1776) }\end{array}$ & Lluna & npk & $\begin{array}{l}\text { Moina minuta } \\
\text { Hansen, } 1899\end{array}$ & Mmin & $\mathrm{pk}$ \\
\hline $\begin{array}{l}\text { Lecane lunaris } \\
\text { (Ehrenberg, 1832) }\end{array}$ & Llun & npk & $\begin{array}{c}\text { Nicsmirnovius cf. } \\
\text { fitzpztricki (Chien, 1970) }\end{array}$ & Nfit & npk \\
\hline
\end{tabular}


Table 1. Continued...

\begin{tabular}{|c|c|c|c|c|c|}
\hline Rotifera & Ab. & $\mathbf{C}$ & Rotifera & Ab. & $\mathbf{C}$ \\
\hline $\begin{array}{l}\text { Lecane monostyla } \\
\text { (Daday, 1897) }\end{array}$ & Lmon & npk & $\begin{array}{c}\text { Phryxura dadayi } \\
\text { Birge, } 1910\end{array}$ & Pdad & npk \\
\hline $\begin{array}{l}\text { Lecane papuana } \\
\text { (Murray, 1913) }\end{array}$ & Lpap & npk & Copepoda & & \\
\hline $\begin{array}{l}\text { Lecane quadridentata } \\
\text { (Ehrenberg, 1832) }\end{array}$ & Lqua & npk & $\begin{array}{c}\text { Argyrodiaptomus furcatus } \\
\text { (Sars, 1901) }\end{array}$ & Afurc & $\mathrm{pk}$ \\
\hline $\begin{array}{l}\text { Lecane scutata } \\
\text { (Harring \& Myers, 1926) }\end{array}$ & Lscut & npk & $\begin{array}{c}\text { Notodiaptomus anisitsi } \\
\text { (Daday, 1905) }\end{array}$ & Nanis & $\mathrm{pk}$ \\
\hline $\begin{array}{l}\text { Lecane signifera } \\
\text { (Jennings, 1896) }\end{array}$ & Lsig & npk & $\begin{array}{c}\text { Notodiaptomus conifer } \\
\text { (Sars, 1901) }\end{array}$ & Nconi & $\mathrm{pk}$ \\
\hline Lecane sp. & Lecsp & & $\begin{array}{l}\text { Notodiaptomus deitersi } \\
\text { (Poppe, 1981) }\end{array}$ & Ndei & $\mathrm{pk}$ \\
\hline $\begin{array}{l}\text { Lecane stenroosi } \\
\text { (Meissner, 1908) }\end{array}$ & Lsten & npk & $\begin{array}{c}\text { Notodiaptomus henseni } \\
\text { (Dahl, 1891) }\end{array}$ & Nhen & pk \\
\hline $\begin{array}{l}\text { Lecane stichea Harring, } \\
1913\end{array}$ & Lstic & npk & $\begin{array}{c}\text { Notodiaptomus iheringi } \\
\text { (Wright, 1935) }\end{array}$ & Niher & $\mathrm{pk}$ \\
\hline $\begin{array}{l}\text { Lepadella acuminata } \\
\text { (Ehrenberg, 1834) }\end{array}$ & Leacu & npk & $\begin{array}{c}\text { Notodiaptomus } \\
\text { spinuliferus } \text { Dussart \& } \\
\text { Matsumura-Tundisi, } 1985\end{array}$ & Nspi & $\mathrm{pk}$ \\
\hline $\begin{array}{l}\text { Lepadella benjamini } \\
\text { Harring, } 1916\end{array}$ & Lben & npk & $\begin{array}{c}\text { Notodiaptomus transitans } \\
\text { (Kiefer, 1929) }\end{array}$ & Ntran & $\mathrm{pk}$ \\
\hline $\begin{array}{l}\text { Lepadella ovalis } \\
\text { (O. F. Müller, 1786) }\end{array}$ & Lova & npk & Notodiaptomus sp. & Nsp & \\
\hline $\begin{array}{l}\text { Lepadella patela } \\
\text { (O. F. Müller, 1786) }\end{array}$ & Lpat & npk & $\begin{array}{c}\text { Mesocyclops longisetus } \\
\text { curvatus } \\
\text { (Thiébaud, 1914) }\end{array}$ & Mlon & npk \\
\hline $\begin{array}{l}\text { Lophocaris salpina } \\
\text { (Ehrenberg, 1834) }\end{array}$ & Lsal & npk & $\begin{array}{c}\text { Mesocyclops meridianus } \\
\text { (Kiefer, 1926) }\end{array}$ & Mmer & $\mathrm{pk}$ \\
\hline $\begin{array}{l}\text { Macrochaetus sericus } \\
\text { (Thorpe, 1893) }\end{array}$ & Mser & npk & Metacyclops sp. & Metsp & \\
\hline $\begin{array}{l}\text { Manfredium eudactylota } \\
\text { (Gallagher, 1957) }\end{array}$ & Meud & npk & Microcyclops sp. & Micsp & \\
\hline $\begin{array}{l}\text { Mytilina ventralis } \\
\text { (Ehrenberg, 1832) }\end{array}$ & Mven & npk & $\begin{array}{c}\text { Paracyclops chiltoni } \\
\text { (Thomson, 1882) }\end{array}$ & Pchil & $\mathrm{npk}$ \\
\hline Notholca sp. & Notsp & & $\begin{array}{c}\text { Thermocyclops decipiens } \\
\text { (Kiefer, 1929) }\end{array}$ & Tdec & $\mathrm{pk}$ \\
\hline $\begin{array}{l}\text { Notomata copeus } \\
\text { Ehrenberg, } 1834\end{array}$ & Ncop & npk & $\begin{array}{c}\text { Thermocyclops minutus } \\
\text { (Lowndes, 1934) }\end{array}$ & Tmin & $\mathrm{pk}$ \\
\hline Notomata sp. & Nsp & & & & \\
\hline
\end{tabular}

Cladoceran richness (Figure 2a) and diversity (Figure 3a) was high in the post-filling phase, with higher values in the main channel. In the pre-filling phase, a decreasing downstream trend of the richness and diversity was verified from site 4 towards site 1 , and lower values also occurred in the tributaries. Rotifer evenness (Figure 3c) in the main channel of the Iguaçu River was similar in the pre- and post-filling phases. However, in the tributaries evenness was higher in the post-filling phase, with a minor variation among sites.

For copepods, only evenness had a significant difference between phases, being higher in the post-filling phase. There was a decrease gradient of evenness downstream
Salto Osório Reservoir, corresponding to the upstream zone in the pre-filling phase (Figure $3 \mathrm{~d}$ ).

The NMDS clearly separated species groups of rotifers and microcrustaceans between pre- and post-filling phases (Figure 4). In both taxa, the pre-filling phase station grouping was less spread than in the post-filling phase indicating a more homogeneous condition in the former. For rotifers (Figure 4a), two convergent solutions were found after 14 tries and the stress was 6.56, showing a pre-filling group with many species of low abundance, in contrast to a post-filling group with few highly abundant species, typical of lentic conditions.

No convergent solutions were found for the microcrustacean data, and the best solution occurred after 
20 tries, with a stress of 7.03 (Figure 4b). The results of richness and abundance analyses were similar for rotifers and microcrustaceans. Typical lotic and littoral cladocerans and copepods were present in the pre-filling phase and were absent in the post-filling phase, when organisms adapted to lentic environments were dominant.

For the environmental variables (Table 3), ANOVA showed significant differences $(p<0.05)$ between the pre- and post-filling phases for water transparency, chlorophyll, alkalinity, and total solids, with high values in the post-filling phase, except for total solids. There were few significant Spearman correlations among rotifer and microcrustacean richness with environmental variables in the pre- and post-filling phases (Table 2). Rotifer richness was positively correlated with water transparency in the post-filling phase, and negatively with microcrustaceans
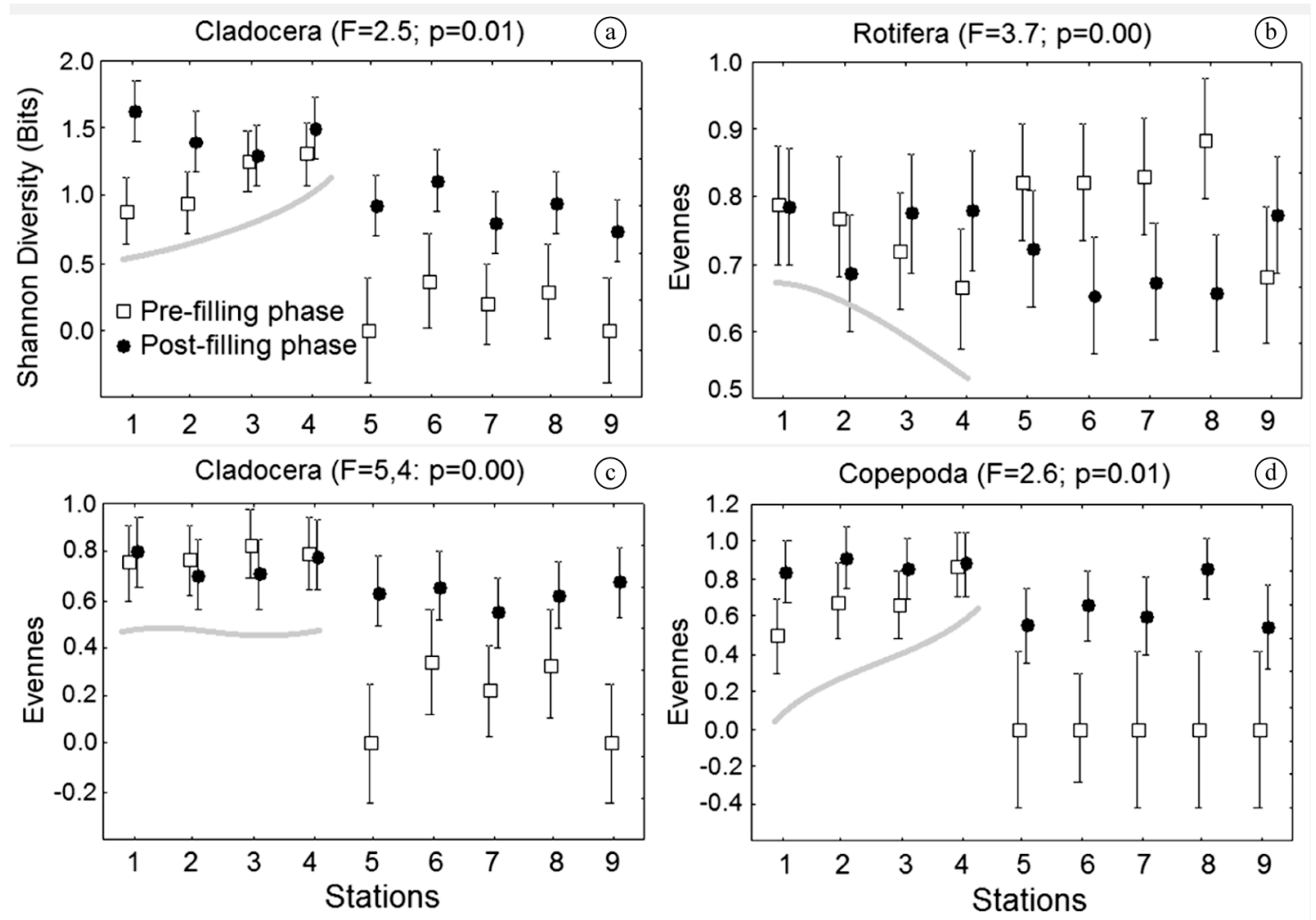

Figure 3. Mean values and standard error for Shannon-Wiener diversity of cladocerans (a), and evenness of rotifers (b), cladocerans (c) and copepods (d), which showed significant interactions in the bifactorial ANOVA.

Table 2. Results of the factorial ANOVA ( $F$ and $p$ ) for richness (S), diversity (H') and evenness (E) of rotifers and microcrustaceans.

\begin{tabular}{|c|c|c|c|c|c|c|}
\hline \multirow{2}{*}{$\begin{array}{c}\text { Groups } \\
\text { Attributes/Effects }\end{array}$} & \multirow{2}{*}{ DF } & \multicolumn{2}{|c|}{ Rotifers } & \multicolumn{2}{|c|}{ Cladocerans } & \multirow{2}{*}{$\frac{\text { Copepods }}{\mathrm{F}}$} \\
\hline & & $\mathbf{F}$ & p & $\mathbf{F}$ & $\mathbf{P}$ & \\
\hline \multicolumn{7}{|l|}{ Richness (S) } \\
\hline Phase & 1 & 98.2 & 0.00 & 129.7 & 0.00 & 20.5 \\
\hline Sites & 8 & 6.3 & 0.00 & 16.3 & 0.00 & 3.8 \\
\hline Phase* Sites & 8 & 3.0 & 0.00 & 2.1 & 0.03 & 0.2 \\
\hline \multicolumn{7}{|l|}{ Diversity } \\
\hline Phase & 1 & 25.1 & 0.00 & 76.1 & 0.00 & 37.4 \\
\hline Sites & 8 & 4.2 & 0.00 & 18.4 & 0.00 & 6.4 \\
\hline Phase* Sites & 8 & 1.1 & 0.30 & 2.5 & 0.01 & 0.7 \\
\hline \multicolumn{7}{|l|}{ Evenness } \\
\hline Phase & 1 & 6.4 & 0.01 & 31.8 & 0.00 & 51.4 \\
\hline Sites & 8 & 0.5 & 0.83 & 11.0 & 0.00 & 8.6 \\
\hline Phase* Sites & 8 & 3.7 & 0.00 & 5.4 & 0.00 & 2.6 \\
\hline
\end{tabular}

* Bold values are significant $(\mathrm{p}<0.05)$. DF $=$ degrees of freedom. 

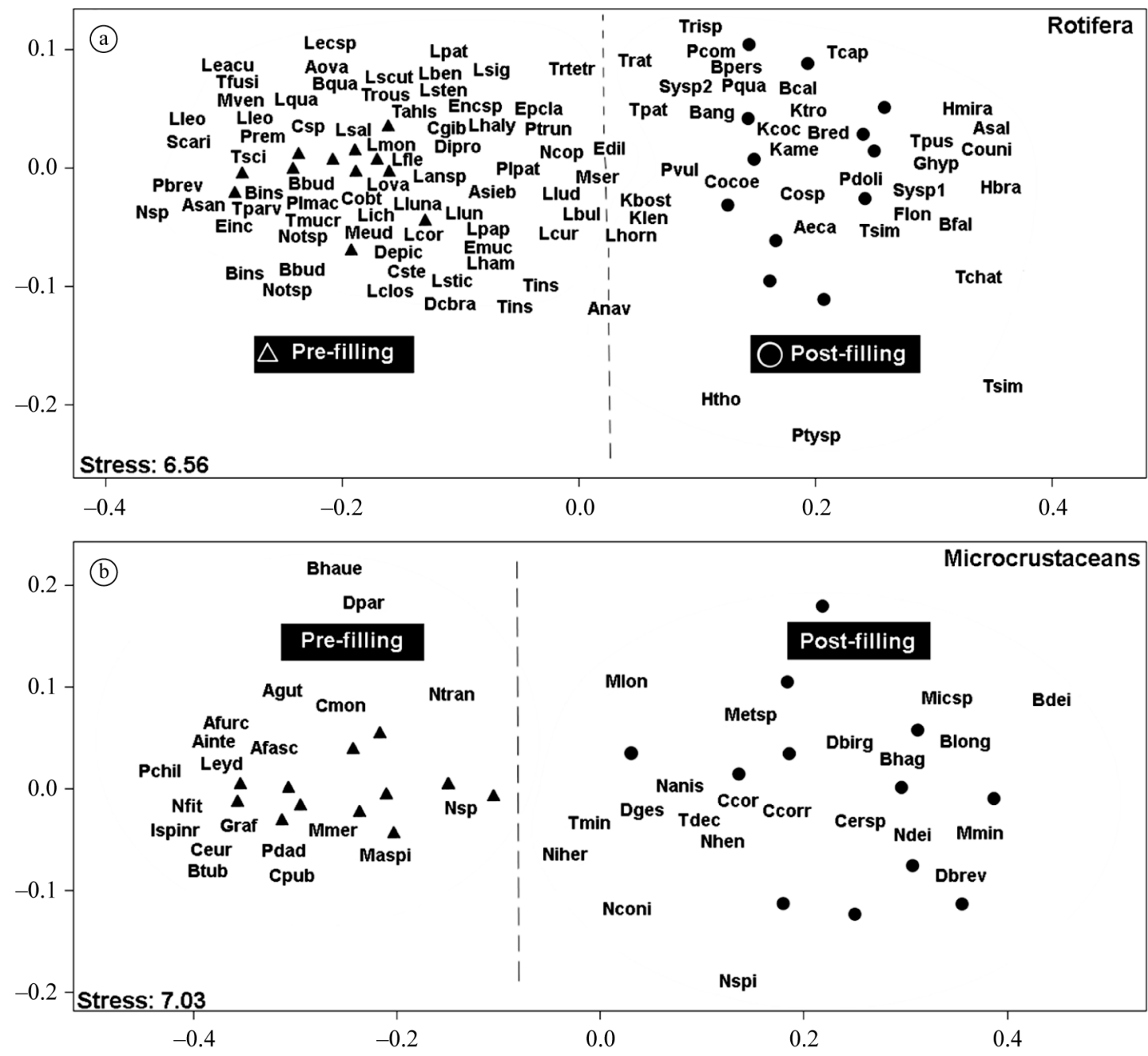

Figure 4. NMDS analyses for the zooplanktonic community between pre- and post-filling phases. (a) Rotifera. (b) Microcrustaceans (Cladocera and Copepoda). For codes see Table 1.

Table 3. Mean values \pm standard deviation of environmental variables, with ANOVA results (in bold are significant differences at $\mathrm{p}<0.05$ level; NS = not significant); and results of Spearman correlation among Rotifera and microcrustacean species richness and environmental variables.

\begin{tabular}{|c|c|c|c|c|c|c|}
\hline \multirow{2}{*}{ Variables } & \multicolumn{2}{|c|}{ Mean \pm SD } & \multicolumn{2}{|c|}{ Rotifera (S) } & \multicolumn{2}{|c|}{ Microcrustaceans (S) } \\
\hline & Pre & Post & Pre & Post & Pre & Post \\
\hline Transparency $(\mathrm{cm})$ & $87.5 \pm 57.78$ & $229.58 \pm 79.41$ & -0.45 & 0.66 & -0.56 & 0.08 \\
\hline Water temperature $\left({ }^{\circ} \mathrm{C}\right){ }^{\mathrm{NS}}$ & $21.29 \pm 2.74$ & $22.62 \pm 1.66$ & 0.20 & -0.08 & 0.15 & -0.60 \\
\hline Chlorophyll $\left(\mu \mathrm{g} . \mathrm{L}^{-1}\right)$ & $1.99 \pm 0.08$ & $2.84 \pm 0.26$ & 0.30 & -0.07 & 0.13 & 0.15 \\
\hline Conductivity $\left(\mu \mathrm{S} . \mathrm{cm}^{-1}\right)^{\mathrm{NS}}$ & $35 \pm 1.35$ & $38.17 \pm 1.90$ & -0.69 & -0.28 & -0.47 & -0.40 \\
\hline Alkalinity (mg.L CaCO3) & $7.67 \pm 2.05$ & $18.06 \pm 2.23$ & -0.37 & -0.22 & -0.37 & 0.41 \\
\hline Dissolved oxygen $\left(\mathrm{mg} \cdot \mathrm{L}^{-1}\right)^{\mathrm{NS}}$ & $7.73 \pm 0.09$ & $7.04 \pm 0.08$ & -0.07 & -0.67 & 0.10 & 0.37 \\
\hline $\mathrm{pH}^{\mathrm{NS}}$ & $7.01 \pm 0.60$ & $7.16 \pm 1.42$ & -0.17 & -0.43 & -0.14 & -0.13 \\
\hline TS (mg.L $\left.\mathrm{L}^{-1}\right)$ & $58.42 \pm 0.29$ & $40.08 \pm 0.57$ & 0.05 & -0.21 & 0.33 & -0.04 \\
\hline TSS $\left(m g . L^{-1}\right){ }^{\mathrm{NS}}$ & $12.23 \pm 23.63$ & $10.46 \pm 9.63$ & 0.34 & 0.31 & 0.57 & 0.05 \\
\hline Total phosphorus (mg.L $\left.\mathrm{L}^{-1}\right)^{\mathrm{NS}}$ & $0.04 \pm 1.08$ & $0.03 \pm 2.39$ & 0.33 & -0.31 & 0.58 & -0.44 \\
\hline Total nitrogen (mg. $\left.\mathrm{L}^{-1}\right)^{\mathrm{NS}}$ & $0.65 \pm 0.45$ & $0.66 \pm 0.56$ & 0.02 & 0.02 & 0.15 & -0.53 \\
\hline
\end{tabular}

In bold are significant correlations at the $\mathrm{p}<0.05$ level. $\mathrm{NS}=$ not significant. 
in the pre-filling phase. Rotifer richness was negatively correlated with conductivity in the pre-filling phase. Rotifers and microcrustaceans were negatively correlated with dissolved oxygen and water temperature, respectively, in the post-filling phase. Microcrustaceans were positively correlated with total suspended solids and total phosphorus in the pre-filling phase.

\section{Discussion}

Detailed rotifers and microcrustaceans species inventories for the Iguaçu River can be found in several studies (Lopes et al., 1997; Ghidini et al., 2009; Serafim-Júnior et al., 2010; Perbiche-Neves et al., 2007, 2012). Among the 140 identified planktonic and non-planktonic species, the littoral Lecanidae (rotifer) and Chydoridae (cladoceran) species contributed significantly to the total richness in the pre-filling phase. In contrast, planktonic forms dominated in the post-filling phase. Similar results were also found in the Corumbá Reservoir in Goiás State (Lansac-Tôha et al., 1999; Bonecker et al., 2001). The number of species identified in our study (140 species) is higher than that identified by Lopes et al. (1997) in Segredo Reservoir (90 species), and by Perbiche-Neves et al. (2012) in Salto Caxias Reservoir (92 species). This may be attributed to the longer period of study and higher sampling effort in nine sampling sites. The effect of filling on the organisms caused an increase of species richness (131 taxa) compared to the pre-impoundment phase (106 taxa), as previously observed in other reservoirs in Brazil for zooplankton (Pedrozo et al., 2012) and for phytoplankton (Bicudo et al., 2006).

Most rotifer species that occurred exclusively in the pre-impoundment phase are littoral and frequently associated with river potamoplankton (Paggi and Jose de Paggi, 1990). The species that occurred exclusively in the post-impoundment phase were mainly planktonic, and were favoured by recently established lentic conditions. Most of the littoral species were found in the backwater sampling sites, with the exception of Platyias quadricornis, which was found in the main channel of the reservoir, probably originating upstream.

The Chydoridae presented the highest number of species among the cladoceran. However, the most frequent cladocerans belonged to the Daphniidae and Bosminidae families, which are commonly found in tropical water bodies (Dumont, 1994). Bosmina hagmanni, Ceriodaphnia cornuta, C. cornuta rigaudi, Daphnia gessneri and Moina minuta were the most frequent species in the pre-and post-filling phases. These species are frequent in the Neotropical region (Dodson and Silva-Briano, 1996; Pinto-Coelho, 1998; Nogueira, 2001; Sendacz et al., 2006; Perbiche-Neves and Nogueira, 2010). Moina reticulata, which only occurred in the pre-filling phase, is endemic to the Neotropical region (Paggi, 1993). After inundation of the reservoir, the emergence of littoral species exclusive to this phase may be explained by upstream export, which was more frequent at the backwater sites. In addition, Bosmina huaroensis was found only in the post-filling phase.
Copepods were clearly favoured by inundation as several species only occurred in the post-impoundment phase, as observed in other studies (Lansac-Tôha et al., 1999). Regarding the most frequent species of Cyclopidae (Cyclopoida), Thermocyclops decipiens is widely distributed in the Neotropical region (Landa et al., 2007; Santos-Wisniewski and Rocha, 2007) and often co-exists with T. minutus (Reid, 1989). However, co-existence was not often observed during this study, nor in the Segredo Reservoir (Lopes et al., 1997), located upstream in the same river. Mesocyclops longisetus and M. meridianus occurred only in the post-filling phase, favoured by the lentic new conditions.

The changes found by other studies (e.g., Garrido and Bozelli, 2000; Pedrozo et al., 2012) were supported by the ANOVA results, which showed significant changes for most ecological attributes of rotifers and microcrustaceans and also for some environmental/limnological variables, considering the interaction between sites and phases. For the tributaries, there was no change in the spatial variation trend, which is different from that observed for the sites located at the main channel of the Iguaçu River, as previously discussed. The NMDS grouped sampling sites, rotifers and microcrustacean species in the pre-filling phase, while the post-filling phase formed a disperse group in the plot graph of this analysis. Non-planktonic species dominated in pre-filling phase, in contrast with reservoir phase, when distinct spatial compartments appeared and caused an aggregated group in the NMDS analyses.

Studies on the spatial distribution of zooplankton from impounded rivers show a reduction in both density and species richness below dam (Unni and Naik, 1997; Mitsuka and Henry, 2002), consistent with the serial discontinuity concept proposed by Ward and Stanford (1983).

The Salto Caxias Reservoir is the fifth largest dam along the Iguaçu River, and the continuous succession of reservoirs has affected the composition of the rotifers and microcrustaceans community in the pre- and postfilling phases of this new reservoir. First, the decreasing gradient of ecological attributes before filling is a result of the drift of organisms from the upstream reservoir (Salto Osorio Reservoir). Second, the rotifers and microcrustaceans species exported downstream from Salto Osorio Reservoir colonised the new reservoir, as shown by NMDS (Perbiche-Neves et al., 2012). Probably the same trend will occur in the sixth new reservoir. Similar results of fast colonisation were found by Garrido and Bozelli (2000) during the filling of another large reservoir in Brazil. The higher species richness in the Iguaçu River compared to tributaries in both phases is probably due to the drift of species downstream from upstream reservoirs and the large size of the river, as occurs in most rivers connected to permanent or temporary bodies of water, from where individuals are exported (Pourriot et al., 1997; Lopes et al., 1997; Viroux, 2002; Perbiche-Neves and Nogueira, 2010; Perbiche-Neves et al., 2012).

Although Rotifera richness was positively correlated with water transparency in the post-filling phase, positive 
effects must have occurred also for microcrustaceans, due to the higher richness after filling the reservoir, consistent with the negative correlation found in the pre-filling phase. Turbidity, total suspended solids (TSS) or transparency can directly affect the organisms, especially microcrustaceans, in rivers and reservoirs (Panarelli et al., 2001; Casanova and Henry, 2004; Perbiche-Neves and Nogueira, 2013), and can be positive if phytoplankton were related to suspended solids or another potential food for these organisms, as found in our data relative to the positive correlation between TSS and microcrustacean richness. Total phosphorus can enhance phytoplankton primary productivity, thus affecting food resources for microcrustaceans. Conductivity seems to affect negatively copepods (Matsumura-Tundisi and Tundisi, 2003), but our results also showed negative effects on rotifers.

The two postulated hypotheses were accepted. We conclude that the formation of a reservoir tends to favour opportunistic coloniser species able to tolerate the new environmental conditions (e.g., increased transparency and chlorophyll content), or those species that easily adapt to the new environmental conditions.

The colonisation process in the Salto Caxias Reservoir has occurred distinctly between the Iguaçu River and tributaries, as shown by the different values presented by the ANOVA, the NMDS results and by comparing with literature about downstream reservoir effects. The rupture in lotic continuity was evident also by the results of the NMDS, separating clearly the homogeneous pre-filling phase and post-filling disperses phases.

\section{Acknowledgements}

We thank Dr. Paulina Barbosa, Dr. Christina Castelo Branco, Dr. Luis Felipe Machado Velho, Dr. Claudia Costa Bonecker and Dr. Barbara Robertson for their valuable suggestions, as well as anonymous referees for suggestions. Funding and logistics were provided by COPEL and CAPES.

\section{References}

BICUDO, D.C., FERRAGUT, C., CROSSETTI, L.O. and BICUDO, C.E.M., 2006. Efeitos do represamento sobre a estrutura da comunidade fitoplanctônica do reservatório de Rosana, baixo rio Paranapanema, Estado de São Paulo. In: M.G. NOGUEIRA, R. HENRY and A. JORCIN. Ecologia de Reservatórios: impactos potenciais, ações de manejo e sistemas em cascatas. São Carlos: Rima, pp. 349-377.

BONECKER, C.C., LANSAC-TÔHA, F.A., VELHO, L.F.M. and ROSSA, D.C., 2001. The temporal distribution pattern of copepods in Corumbá Reservoir, State of Goias, Brazil. Hydrobiologia, vol. 453, pp. 375-384. http://dx.doi.org/10.1023/A:1013142101266.

CASANOVA, S.M. and HENRY, R., 2004. Longitidunal distribution of Copepoda populations in the transition zone of Paranapanema River and Jurumirim Reservoir (São Paulo, Brazil) and interchange with two lateral lakes. Brazilian Journal of Biology $=$ Revista Brasileira de Biologia, vol. 64, no. 1, pp. 11-26. http://dx.doi. org/10.1590/S1519-69842004000100003. PMid:15195360.
DODSON, S.I. and SILVA-BRIANO, M., 1996. Crustacean zooplankton species richness and associations in Reservoirs and pounds of Aguascalientes State, Mexico. Hydrobiologia, vol. 325, no. 2, pp. 163-172. http://dx.doi.org/10.1007/BF00028277.

DUMONT, H.J., 1994. On the diversity of the Cladocera in the tropics. Hydrobiologia, vol. 272, no. 1-3, pp. 27-38. http://dx.doi. org/10.1007/BF00006510.

GARRIDO, A.V. and BOZELLI, R.L., 2000. The study of zooplankton during the filling of the Serra da Mesa Reservoir, Tocantins River (GO, Brazil). Verhandlungen der Internationalen Vereinigung für Theoretische und Angewandte Limnologie, vol. 27, pp. 2875-2878.

GHIDINI, A.R., SERAFIM-JÚNIOR, M., PERBICHE-NEVES, G. and BRITO, L., 2009. Distribuição de Cladocera (Crustacea) em um reservatório eutrófico na bacia do alto rio Iguaçu, Paraná, Brasil. Pan-American Journal of Aquatic Sciences, vol. 4, pp. 294-305.

KOSTE, W., 1978. Rotatoria die Rodertiere itteleuropas begtündet von Max Voigt - Monogononta. 2. Auflage neubearbeitet von Walter Koste. Berlin: Gebrüder Borntraeger, vol. 1-2.

LANDA, G.G., BARBOSA, F.A.R., RIETZLER, A.C. and BARBOSA, P.M., 2007. Thermocyclops decipiens (Kiefer, 1929) (Copepoda, Cyclopoida) as Indicator of Water Quality in the State of Minas Gerais, Brazil. Brazilian Archives of Biology and Technology, vol. 50, no. 4, pp. 695-705. http://dx.doi.org/10.1590/ S1516-89132007000400015.

LANSAC-TÔHA, F.A., VELHO, L.F.M. and BONECKER, C.C., 1999. Estrutura da comunidade zooplanctônica antes e após a formação do reservatório de Corumbá-GO. In: R. HENRY. Ecologia de reservatórios: estrutura, função e aspectos sociais. Botucatu: Universidade Estadual Paulista/Fundibio, pp. 347-374.

LIMA, A.F. and LANSAC-TÔHA, F.A., VELHO, L.F.M. and BINI, L.M., 1998. Environmental influence on planktonic cladocerans and copepods in the floodplain of the Upper River Paraná, Brazil. Studies on Neotropical Fauna and Environment, vol. 33, no. 1, pp. 188-196.

LOPES, R.M., LANSAC-TÔHA, F.A., VALE, R. and SERAFIMJÚNIOR, M., 1997. Comunidade zooplanctônica do reservatório de Segredo. In: A.A. AGOSTINHO and L.C. GOMES. Reservatório de segredo: bases ecológicas para o manejo. Maringá: Eduem, pp. 39-60.

MACKRETH, F.J.H.; HERON, J. and TALLING, J. F., 1978. Water analysis: some revised methods for limnologist. Kendall: Titus Wilson e Sons LTD. 120 p. Freshwater Biological Association/ Scientific Publication, no. 36.

MARZOLF, R.G., 1990. Reservoirs as environments for zooplankton. In: K.M. THORNTON, L.B. KIMMEL and F.E. PAYNE. Reservoir limnology: ecological perspectives. New York: John Wiley and Sons, pp. 195-208.

MATSUMURA-TUNDISI, T. and TUNDISI, J.G., 2003. Calanoida (Copepoda) species composition changes in the reservoirs of São Paulo State (Brazil) in the last twenty years. Hydrobiologia, vol. 504, no. 1-3, pp. 215-222. http://dx.doi. org/10.1023/B:HYDR.0000008521.43711.35.

MATSUMURA-TUNDISI, T., TUNDISI, J.G., SAGGIO, A., OLIVEIRA-NETO, A.L. and ESPINDOLA, E.G., 1991. Limnology of Samuel Reservoir (Brazil, Rondonia) in the filling phase. Verhandlungen der Internationalen Vereinigung für Theoretische und Angewandte Limnologie, vol. 24, pp. 1482-1488. 
MITSUKA, P.M. and HENRY, R., 2002. The fate of copepod populations in the Paranapanema River (São Paulo, Brazil), downstream from the Jurumirim Dam. Brazilian Archives of Biology and Technology, vol. 45, no. 4, pp. 479-490. http://dx.doi. org/10.1590/S1516-89132002000600012.

NOGUEIRA, M.G., 2001. Zooplankton composition dominance and abundance as indicators of environmental compartmentalization in Jurumirim Reservoir (Paranapanema River), São Paulo, Brazil. Hydrobiologia, vol. 455, no. 1/3, pp. 1-18. http://dx.doi. org/10.1023/A:1011946708757.

NOGUEIRA, M.G., REIS-OLIVEIRA, P.C. and BRITTO, Y.T., 2008. Zooplankton assemblages (Copepoda and Cladocera) in a cascade of reservoirs of a large tropical river (SE Brazil). Limnetica, vol. 27, no. 1, pp. 151-170.

PAGGI, J.C. and JOSÉ DE PAGGI, S., 1990. Zooplâncton de ambientes lóticos e lênticos do rio Paraná Médio. Acta Limnologica Brasiliensia, vol. 3, pp. 685-719.

PAGGI, J.C., 1993. Preliminary analisys of geographic distribution of sudamerican cladocerans. In: A. BOLTOVSKOY and H.L. LOPEZ. Conference on Limnology, November 1991, La Plata, Argentina. La Plata: Instituto de Limnología Dr RA Ringuelet, pp. 108-113.

PANARELLI, E.A., NOGUEIRA, M.G. and HENRY, R., 2001. Short-term variability of copepod abundance in Jurumirim Reservoir, São Paulo, Brazil. Brazilian Journal of Biology = Revista Brasileira de Biologia, vol. 61, no. 4, pp. 577-598. http:// dx.doi.org/10.1590/S1519-69842001000400007. PMid:12071314.

PEDROZO, C.S., SCHNECK, F., SCHWARZBOLD, A. and FARIAS, R.N., 2012. Respostas da comunidade zooplanctônica à formação do reservatório de Dona Francisca, Rio Grande do Sul, Brasil. Iheringia Série Zoologia, vol. 102, no. 2, pp. 142-149. http://dx.doi.org/10.1590/S0073-47212012000200005.

PERBICHE-NEVES, G. and NOGUEIRA, M.G., 2010. Multidimensional effects on cladoceran (Crustacea, Anomopoda) assemblages in two cascade reservoirs (SE - Brazil). Lakes and Reservoirs: Research and Management, vol. 15, no. 2, pp. 151164. http://dx.doi.org/10.1111/j.1440-1770.2010.00429.x.

PERBICHE-NEVES, G., FERREIRA, R.A.R. and NOGUEIRA, M.G., 2011. Phytoplankton structure in two contrasting cascade reservoirs (Paranapanema River, Southeast Brazil). Biologia Section Botany, vol. 66, no. 6, pp. 967-976.

PERBICHE-NEVES, G., SERAFIM-JÚNIOR, M., GHIDINI, A.R. and BRITO, L., 2007. Spatial and temporal distribution of Copepoda (Cyclopoida and Calanoida) of an eutrophic reservoir in the basin of upper Iguaçu River, Paraná, Brazil. Acta Limnologica Brasiliensia, vol. 19, pp. 393-406.

PERBICHE-NEVES, G., SERAFIM-JÚNIOR, M., PORTINHO, J.L., SHIMABUKURO, E.M., GHIDINI, A.R. and BRITO, L., 2012. Effects of atypical rainfall on lotic zooplankton: comparing downstream of a reservoir and tributaries with free stretches. Tropical Ecology, vol. 53, pp. 149-169.

PERBICHE-NEVES, N. and NOGUEIRA, M.G., 2013. Reservoir design and operation: effects on aquatic biota - a case study of planktonic copepods. Hydrobiologia, vol. 707, no. 1, pp. 187-198. http://dx.doi.org/10.1007/s10750-012-1425-1.

PIELOU, E.C., 1984. The interpretation of ecological data. New York: John Wiley. 263 p.

PINTO-COELHO, R.M., 1998. Effects of eutrophication on seasonal patterns of mesozooplankton in a tropical Reservoir: a 4-year study in Pampulha Lake, Brazil. Freshwater Biology, vol. 40, no. 1, pp. 159-173. http://dx.doi.org/10.1046/j.13652427.1998.00327.x.

POURRIOT, R., ROUGIER, C. and MIQUELIS, A., 1997. Origin and development of river zooplankton: example of the Marne. Hydrobiologia, vol. 345 , no. $2 / 3$, pp. 143-148. http://dx.doi. org/10.1023/A:1002935807795.

R CORE DEVELOPMENT TEAM, 2012 [viewed 10 December 2012]. A language and environment for statistical computing [online]. Vienna: R Foundation for Statistical Computing. Available from: http://www.R-project.org

REID, J.W., 1989. The distribution of species of the genus Thermocyclops (Copepoda, Cyclopoida) in the western hemisphere, with description of T. parvus, new species. Hydrobiologia, vol. 175, no. 2, pp. 149-179. http://dx.doi.org/10.1007/BF00765125.

RIBEIRO FILHO, R.A., PETRERE JUNIOR, M., BENASSI, S.F. and PEREIRA, J.M.A., 2011. Itaipu reservoir limnology: eutrophication degree and the horizontal distribution of its limnological variables. Brazilian Journal of Biology $=$ Revista Brasileira de Biologia, vol. 71, no. 4, pp. 889-902. http://dx.doi. org/10.1590/S1519-69842011000500010.

SAMPAIO, E.V., ROCHA, O., MATSUMURA-TUNDISI, T. and TUNDISI, J.G., 2002. Composition and abundance of zooplankton in the limnetic zone of seven reservoirs of the Paranapanema River, Brazil. Brazilian Journal of Biology $=$ Revista Brasileira de Biologia, vol. 62, no. 3, pp. 525-545. http://dx.doi.org/10.1590/ S1519-69842002000300018. PMid:12530191.

SANTOS, G.M., 1995. Impactos da hidrelétrica samuel sobre as comunidades de peixes do Rio Jamari (Rondônia, Brasil). Acta Amazonica, vol. 25, pp. 247-280. http://dx.doi.org/10.1590/180943921995253280 .

SANTOS-WISNIEWSKI, M.J. and ROCHA, O., 2007. Spatial distribution and secondary production of Copepoda in a tropical reservoir: Barra Bonita, SP, Brazil. Brazilian Journal of Biology = Revista Brasileira de Biologia, vol. 67, no. 2, pp. 223-233. http:// dx.doi.org/10.1590/S1519-69842007000200007. PMid:17876432.

SARTORI, L.P., NOGUEIRA, M.G., HENRY, R. and MORETTO, E.M., 2009. Zooplankton fluctuations in Jurumirim Reservoir (São Paulo, Brazil): a three-year study. Brazilian Journal of Biology = Revista Brasileira de Biologia, vol. 69, no. 1, pp. 1-18. http:// dx.doi.org/10.1590/S1519-69842009000100002. PMid:19347141.

SEGERS, H., 1995. Rotifera: the Lecanidae (Monogononta). The Hague: SPB Academics, $226 \mathrm{p}$. Guides to the identification of the microinvertebrates of the continental waters of the world, vol. 2.

SENDACZ, S., CALEFFI, S. and SANTOS-SOARES, J., 2006. Zooplankton biomass of reservoirs in different trophic conditions in the State of São Paulo, Brazil. Brazilian Journal of Biology = Revista Brasileira de Biologia, vol. 66, no. 1B, pp. 337-350. http:// dx.doi.org/10.1590/S1519-69842006000200016. PMid:16710526.

SERAFIM JUNIOR, J.R.M., LANSAC-TÔHA, F.A., PAGGI, J.C., VELHO, L.F.M. and ROBERTSON, B., 2003. Cladocera fauna composition in a river-lagoon system of the Upper Paraná River floodplain, with a new record for Brazil. Brazilian Journal of Biology $=$ Revista Brasileira de Biologia, vol. 63, no. 2, pp. 349-356. http://dx.doi.org/10.1590/S1519-69842003000200020. PMid:14509857.

SERAFIM-JUNIOR, M., PERBICHE-NEVES, G., BRITO, L., GHIDINI, A.R. and CASANOVA, S.M.C., 2010. Variação espaço- 
temporal de Rotíferos em um reservatório eutrofizado no sul do Brasil. Iheringia série. Zoologia, vol. 100, no. 3, pp. 233-241.

STATSOFT INC, 2002. Statistica: data analysis software system: version 6.0. Tulsa: STATSOFT INC.

STRASKRABA, M. and TUNDISI, J.G., 1999. Reservoir ecosystem functioning: theory and application. In: J.G. TUNDISI and M. STRASKRABA. Theoretical Reservoir ecology and its applications. São Carlos: International Institute of Ecology, pp. 565-583.

STRICKLAND, J.D. and PARSONS, T.R., 1960. A manual or sea water analisys. Bulletin Fisheries Research Board of Canada, vol. 125 , pp. 1-185.

TALLING, J.F. and DRIVER, D., 1963. Some problems in the estimation of chlorophyll a in phytoplankton. In: Proceendings on conference of primary productivity measurements in marine and freshwater, 21 August-06 September 1961, Manoa, Hawaii. Hawaii: USAEE, pp. 142-146.

UNNI, K.S. and NAIK, L., 1997. Distribution and ecology of the zooplankton in the headwaters of the tropical River Narmada. International Journal of Ecology and Environmental Sciences, vol. 23, pp. 1-16.
VAN DEN BRINK, F.W.B., VAN KATWIJK, M.M. and VAN DER VELDE, G., 1994. Impact of hidrology and phyto and zooplankton community composition in floodplain lakes along the Lower Rhine and Meuse. Journal of Plankton Research, vol. 16, no. 4, pp. 351-373. http://dx.doi.org/10.1093/plankt/16.4.351.

VELHO, L.F.M., LANSAC-TÔHA, F.A., BONECKER, C.C., BINI, L.M. and ROSSA, D.C., 2001. The longitudinal distribution of copepods in Corumbá Reservoir, State of Goiás, Brazil. Hydrobiologia, vol. 453, pp. 385-391. http://dx.doi. org/10.1023/A:1013194118104.

VIROUX, L., 2002. Seasonal and longitudinal aspects of microcrustacean (Cladocera, Copepoda) dynamics in a lowland River. Journal of Plankton Research, vol. 26, pp. 281-292.

WARD, J.V. and STANFORD, J.A., 1983. The serial discontinuity concept of lotic ecosystems. In: T.D. FONTAINE and S.M. BARTELL. Dynamics of lotic ecosystems. Michigan: The Ann Arbor Science Publishers, pp. 29-42.

WARD, J.V., 1989. The four-dimensional natures of lotic ecosystems. Journal of the North American Benthological Society, vol. 8, no. 1, pp. 2-8. http://dx.doi.org/10.2307/1467397. 